\title{
A HOLONOMY PROOF OF THE POSITIVE CURVATURE OPERATOR THEOREM ${ }^{1}$
}

\author{
W. A. POOR
}

\begin{abstract}
ABstracr. Extending work of Bochner-Yano and M. Berger, D. Meyer proved that if the curvature operator of a compact, oriented, Riemannian manifold $\boldsymbol{M}$ has positive eigenvalues, then $M$ is a rational homology sphere. Here a proof is given using Chern's holonomy formula for the Laplacian on $\boldsymbol{M}$; for completeness, a quick proof of Chern's formula is included.
\end{abstract}

Chern [3] showed that the Hodge theory of compact Kähler manifolds is a special case of a general phenomenon; by reinterpreting Weizenböck's formula for the Laplacian of a Riemannian manifold in terms of the holonomy group of the manifold, he was able to simplify many of the classical cohomology calculations on Kähler manifolds, while simultaneously exhibiting other geometric structures for which similar theorems hold.

In this note Chern's formula is used to simplify the proof of the following result.

THEOREM. If $M^{n}$ is an oriented, compact Riemannian manifold with positive definite curvature operator, then $M$ is a rational homology sphere.

Bochner and Yano [2], [7] proved that if the eigenvalues of the curvature operator of $M$ lie in the interval $\left[\frac{1}{2}, 1\right]$, then by Weizenböck's formula,

$$
\int_{M}\langle\Delta \mu, \mu\rangle>\frac{r(n-2 r+1)}{2} \int_{M}\|\mu\|^{2}
$$

for an $r$-form $\mu$ on $M$. Thus $H^{r}(M ; \mathbf{R})$ is zero for $r=1,2, \ldots, n-1$.

Berger [1] observed that for a form $\mu$ of degree 2, the integral over $M$ of $\langle\Delta \mu, \mu\rangle$ could be estimated from below in terms of the minimum $\lambda$ of the eigenvalues of the curvature operator; this proved that $H^{2}(M ; R)$ is zero if the curvature operator of $M$ is positive definite. He then asked whether this hypothesis implies the vanishing of $H^{r}(M ; \mathbf{R})$ for $0<r<n$.

This question was answered affirmatively by Meyer [6]. Subsequently, Gallot and Meyer [4] obtained the sharp estimate

$$
\int_{M}\langle\Delta \mu, \mu\rangle \geqslant \lambda r(n-r) \int_{M}\|\mu\|^{2}
$$

for all $r$-forms on $M$, which makes the proof of the theorem completely obvious.

Received by the editors January 25, 1979 and, in revised form, August 14, 1979.

AMS (MOS) subject classifications (1970). Primary 53C20.

Key words and phrases. Eigenvalue of curvature operator, Laplacian, rational homology sphere, holonomy algebra.

${ }^{1}$ This work was supported in part by NSF grant MCS72 05055 A04 while the author was at the Institute for Advanced Study. 
In this note, inequality (1) will follow from Chern's holonomy formula for the Laplacian by comparison of $\langle\Delta \mu, \mu\rangle$ with the Laplacian on the sphere of curvature $\lambda$. For completeness, a quick derivation of Chern's formula is included; cf. also Weil [8].

The author would like to thank Sylvain Gallot and Daniel Meyer, as well as the referee, for informing him of the thesis of Maillot [5], which contains calculations quite similar to some of those in this note.

1. Chern's formula for the Laplacian. Fix an $r$-form $\mu$ on $M$. Given a point $p$ in $M$, vectors $v_{1}, \ldots, v_{r}$ in $M_{p}$, and an orthonormal basis $\left\{e_{j}\right\}$ for $M_{p}$, define

$$
\Re_{\mu}\left(v_{1}, \ldots, v_{r}\right):=\sum_{i=1}^{n} \sum_{j=1}^{r}\left(R\left(e_{i}, v_{j}\right) \mu\right)\left(v_{1}, \ldots, v_{j-1}, e_{i}, v_{j+1}, \ldots, v_{r}\right) .
$$

Weizenböck's formula states that

$$
\langle\Delta \mu, \mu\rangle=\frac{1}{2} \Delta\|\mu\|^{2}+\|\nabla \mu\|^{2}+\left\langle\Re_{\mu}, \mu\right\rangle .
$$

If $u$ and $v$ are tangent vectors on $M$ at $p$, then it is well known that

$$
R(u, v) \mu=-\mu \circ R(u, v)={ }^{t} R(u, v)(\mu)
$$

where we use the natural extension of $R(u, v) \in \operatorname{End}\left(M_{p}\right)$ to a derivation of $\Lambda_{r} M_{p}$, and then take the transpose to get an element of $\operatorname{End}\left(\Lambda_{r} M_{p}^{*}\right)$.

Now suppose that the holonomy group of $M$ is the Lie subgroup $G$ of $S O(n)$. The holonomy algebra $g\left(M_{p}\right) \subset o\left(M_{p}\right)$ can be embedded into $\Lambda_{2} M_{p}$ by

$$
\langle X, u \wedge v\rangle_{\Lambda_{2} M_{p}}:=\langle X(v), u\rangle_{M_{p}}, \quad X \in g\left(M_{p}\right), u, v \in M_{p} .
$$

Let $\left\{X_{\alpha}\right\}$ be an orthonormal basis for $g\left(M_{p}\right)$; because of the equality $R(u, v)=$ $\Sigma_{\alpha}\left\langle R(u, v), X_{\alpha}\right\rangle X_{\alpha}=\Sigma_{\alpha}\left\langle R\left(X_{\alpha}\right) v, u\right\rangle X_{\alpha}$,

$$
\begin{aligned}
\Re_{\mu}\left(v_{1}, \ldots, v_{r}\right) & =-\sum_{i=1}^{n} \sum_{j=1}^{r} \sum_{\alpha}\left\langle R\left(X_{\alpha}\right) v_{j}, e_{i}\right\rangle^{t} X_{\alpha}(\mu)\left(v_{1}, \ldots, v_{j-1}, e_{i}, v_{j+1}, \ldots, v_{r}\right) \\
& =-\sum_{\alpha}\left({ }^{t} R\left(X_{\alpha}\right)^{t} X_{\alpha} \mu\right)\left(v_{1}, \ldots, v_{r}\right) .
\end{aligned}
$$

Thus

$$
\langle\Delta \mu, \mu\rangle(p)=\left(\frac{1}{2} \Delta\|\mu\|^{\dot{2}}+\|\nabla \mu\|^{2}\right)(p)+\sum_{\alpha}\left\langle{ }^{t} X_{\alpha} \mu,{ }^{t} R\left(X_{\alpha}\right) \mu\right\rangle
$$

since $R\left(X_{\alpha}\right)$ is skew-symmetric; this is Chern's formula.

2. Proof of the positive curvature operator theorem. Assume the notation of $\S 1$. Let all the eigenvalues of $R$ be greater than or equal to $\lambda>0$. Fix $p \in M$ and choose an orthonormal basis $\left\{X_{\alpha}\right\}$ for $o\left(M_{p}\right) \simeq \Lambda_{2} M_{p}$. Since $\left\langle R\left(X_{\alpha}\right), X_{\alpha}\right\rangle>\lambda>0$ for all $\alpha$,

$$
\sum_{\alpha}\left\langle{ }^{t} R\left(X_{\alpha}\right) \mu,{ }^{t} X_{\alpha} \mu\right\rangle>\lambda \sum_{\alpha}\left\|^{t} X_{\alpha} \mu\right\|^{2} .
$$

It also follows that the holonomy algebra $g\left(M_{p}\right)$ equals $o\left(M_{p}\right)$, which allows us to calculate the right-hand side of (3) on any appropriate space. Isometrically identify $M_{p}$ with the tangent space $S_{q}^{n}$ at some point $q$ in the $n$-sphere $S^{n} ; \mu_{p}$ corresponds to 
the value $\eta_{q}$ of some $r$-form $\eta$ on $S^{n}$. By [7, §4.1], $\Delta \eta=-\operatorname{div} \nabla \eta+r(n-r) \eta$, so

$$
\left\|^{t} X_{\alpha} \mu_{p}\right\|^{2}=\langle\Delta \eta+\operatorname{div} \nabla \eta, \eta\rangle(q)=r(n-r)\left\|\eta_{q}\right\|^{2}=r(n-r)\left\|\mu_{p}\right\|^{2} .
$$

Thus,

$$
\langle\Delta \mu, \mu\rangle>\frac{1}{2} \Delta\|\mu\|^{2}+\|\nabla \mu\|^{2}+\lambda r(n-r)\|\mu\|^{2} .
$$

Integration over $M$ now yields inequality (1).

\section{REFERENCES}

1. M. Berger, Sur les variétés à opérateur de courbure positif, C. R. Acad. Sci. Paris Sér A-B 253 (1961), 2832-2834.

2. S. Bochner and K. Yano, Tensor-fields in non-symmetric connections, Ann. of Math. (2) 56 (1952), 504-519.

3. S. S. Chern, On a generalization of Kähler geometry, Algebraic Geometry and Topology, Sympos. in honor of S. Lefschetz, Princeton Univ. Press, Princeton, N. J., 1959, pp. 103-121.

4. S. Gallot and D. Meyer, Opérateur de courbure et Laplacien des formes différentielles d'une variété riemannienne, J. Math. Pures Appl. 54 (1975), 259-284.

5. H. Maillot, Sur les variétés riemanniennes à opérateur de courbure pur, C. R. Acad. Sci. Paris Sér. A-B 278 (1974), $1127-1130$.

6. D. Meyer, Sur les variétés riemanniennes à opérateur de courbure positif, C. R. Acad. Sci. Paris Sér. A-B 272 (1971), 482-485.

7. K. Yano and S. Bochner, Curoature and Betti mumbers, Ann. of Math. Studies, no. 32, Princeton Univ. Press, Princeton, N. J., 1953.

8. A. Weil, Un théorème fondamental de Chern en géométrie riemannienne, Séminaire Bourbaki, 14 e année, 1961/62, no. 239.

School of MAthematics, InstTtute for AdVanced Study, Princeton, New Jersey 08540

Current address: Department of Mathematical Sciences, Rensselaer Polytechnic Institute, Troy, New York 12181 